\title{
Estamos todos juntos: el cierre de la Escuela Rural desde la perspectiva de los niños'
}

\author{
Carmen Gloria Núñez" \\ Mónica Peñall! \\ Francisco Cubillos" \\ Héctor Solorzall
}

I- Los datos fueron producidos dentro del Proyecto Fondecyt 11110317 Cuando la escuela rural se cierra: procesos psicosociales a nivel comunitario a partir del cierre y fusión de escuelas rurales en la sexta región, financiado por Conicyt, Chile, 2011 - 2013. Posteriormente, fueron sometidos a revisión y a un nuevo análisis a partir de la experiencia con niñas y niños en el Proyecto DI 037.441/2015 financiado por la Pontificia Universidad Católica de Valparaíso.

II- Pontificia Universidad Católica de Valparaíso, Valparaíso, Chile.

Contactos: carmen.nunez@pucv.cl;

fcoj.cubillos@gmail.com;

allkun.capsi@gmail.com

III- Universidad Diego Portales, Santiago, Chile. Contacto: monica.pena@udp.cl

\section{Resumen}

El cierre sistemático de las escuelas rurales es un fenómeno que viene desarrollándose desde hace más de una década en Chile. Los resultados que se presentan se originan en el contexto de nuestra línea de investigación sobre el cierre de escuelas rurales municipales y sus consecuencias en las relaciones sociales entre las comunidades escolares y locales. En este trabajo presentamos la perspectiva particular de las niñas y niños de una escuela básica rural multigrado respecto del aviso de cierre del centro, para lo cual se desarrolló una metodología de taller con uso de herramientas gráficas, que incorpora algunos elementos del Grupo de Discusión. Los resultados muestran que las niñas y niños valoran sentirse parte de un grupo socialmente cohesionado y que esto constituye un capital social acumulado que es exclusivo de esa escuela, lo cual no es transferible a otra. Asimismo, las niñas y niños interpretan el posible cierre escolar como una medida más dentro de otras transformaciones del sector, en el cual el rol del Municipio ha sido privilegiar intereses foráneos por sobre los de los habitantes locales.

\section{Palabras clave}

Cierre de escuelas - Escuela rural - Investigación social con niños - Cohesión social - Capital social. 


\title{
We are all together: the closing of the rural school from the children's perspective'
}

\author{
Carmen Gloria Núñez" \\ Mónica Peñall! \\ Francisco Cubillos" \\ Héctor Solorzall
}

\begin{abstract}
The systematic closing of rural schools is a phenomenon that has developed for over a decade in Chile. The results presented herein originated from our research on the closing of municipal rural schools and its impact on the social relations between the school and local communities. In this paper we present the particular perspective of the children of a rural multigrade primary school regarding the announcement of the closure of the school, for which a workshop methodology was developed with the use of graphical tools, incorporating some elements of a discussion group. Results show that children appreciate the feeling that they are part of a socially cohesive group and that this is an accumulated capital, which is unique to that school and is not transferable to another. The children also interpret the possible school closing as one more step in other transformations in the sector, in which the municipality has favored foreign interests over those of the locals.
\end{abstract}

\section{Keywords}

School closing - Rural school - Social research with children Social cohesion - Social capital

\footnotetext{
I- Data were produced under Proyecto Fondecyt 11110317 Cuando la escuela rural se cierra: procesos psicosociales a nivel comunitario a partir del cierre y fusión de escuelas rurales en la sexta región, funded by Conicyt, Chile, 2011 - 2013. Later on, they were reviewed and analysed again from the perspective of the children in project DI 037.441/2015 funded by Pontificia Universidad Católica de Valparaíso.

II- Pontificia Universidad Católica de Valparaíso, Valparaíso, Chile.

Contacts: carmen.nunez@pucv.cl;

fcoj.cubillos@gmail.com;

allkun.capsi@gmail.com

III- Universidad Diego Portales, Santiago, Chile.

Contact: monica.pena@udp.cl
} 


\section{Introducción}

Los resultados que se presentan se originan en el contexto de nuestra investigación sobre el cierre de escuelas rurales municipales en Chile y sus consecuencias en las relaciones sociales entre las comunidades escolares y locales. El cierre sistemático de las escuelas rurales es un fenómeno que viene desarrollándose desde hace más de una década en Chile. Según datos del Ministerio de Educación de Chile (MINEDUC), 969 escuelas básicas rurales municipales han dejado de funcionar entre el año 2000 y el 2015². Este cierre masivo de escuelas se relaciona con varios factores, pero principalmente obedece a una racionalidad neoliberal que ha organizado la política educativa en las últimas décadas en Chile (CORNEJO, 2006). El sistema escolar se ha descentralizado y privatizado, a través del sistema del voucher o subvención a la demanda, donde las escuelas compiten entre ellas, dado que se espera que las familias elijan la mejor la escuela para sus hijos. Este sistema ha provocado una enorme segregación entre las escuelas pagadas y las públicas (HSEIH; URQUIOLA, 2002).

En este escenario, este estudio se interesó por los intersticios en donde se encuentran las políticas neoliberales en Chile y las subjetividades que éstas movilizan en los contextos rurales. Con el fin de conocer las consecuencias psicosociales en la comunidad escolar y local del cierre de la escuela rural, se desarrolló una investigación etnográfica con un estudio de caso en torno a una escuela bajo aviso de cierre, en la cual participaron actores municipales, profesores, madres, padres, niñas y niños.

En este artículo queremos concentrarnos específicamente en el grupo de niñas y niños de la escuela estudiada. Nuestro interés fue conocer sus formas particulares de significar la escuela y el lugar que ésta ocupa en la comunidad, relevando la especificidad de la

1- Cálculo de los autores en base a datos publicados por MINEDUC. experiencia infantil en el caso estudiado. Para ello fue necesario diseñar una herramienta metodológica que permitiera la expresión de las niñas y niños en torno al posible cierre de la escuela y sus consecuencias en la cohesión social. Esta decisión fue tomada en tanto no existen muchos estudios latinoamericanos que se concentren en la perspectiva de los niños, dado que se tiende a pensar que estos no son miembros de la comunidad cultural o lingüística a las que pertenecen, ya sea porque se les supone inmadurez lingüística y/o absoluta falta de agencia política. La comprensión de niñez sobre la que se basa esta investigación se sale de las perspectivas más tradicionales psicológicas que ponen a los niños en una línea de desarrollo y madurez, siendo más cercana a los nuevos estudios sociales de la infancia que los consideran sujetos sociales (JAMES; JAMES, 2004; 2008). En este trabajo entonces analizaremos primero el rol de las escuelas como fundamentales en la constitución de las comunidades donde se encuentran, para luego justificar metodológicamente la importancia de indagar las percepciones de los niños, y finalmente mostrar los resultados de este trabajo etnográfico.

\section{La escuela rural y su rol en la comunidad: capital social y cohesión} en el contexto de la nueva ruralidad

Las escuelas tienen un rol fundamental en la constitución de una comunidad. No son solamente microempresas que entregan un servicio: son parte fundamental del tejido social y comunitario de los territorios donde se insertan. Las escuelas brindan una serie de elementos en la vida social de los sujetos, entre ellos el llamado capital social.

Las escuelas públicas proporcionan el capital social específico en la comunidad, principalmente a través de las redes que los padres establecen. Los propios niños también adquieren una red de amigos de las 
escuelas. Los compañeros de escuela son, sin duda, el beneficio del capital social que dura más tiempo que el período de asistencia a la escuela. (FISCHEL, 2006, p. 8).

Fischel no sólo está mencionando el poder de las redes de contactos, sino fundamentalmente el poder de la asociación comunitaria. La perspectiva de capital social de Fischel es más coherente con la de Coleman (1988) que con la de Bourdieu (1985 apud RAMÍREZ, 2005) que supone al capital social una relación mucho más estrecha con la clase social. La visión de Coleman da cuenta de la relación de los individuos con la estructura, y distinguirá las obligaciones y expectativas de los individuos, las normas y sanciones y el potencial de información disponible (COLEMAN, 1988).

El mismo autor considera que el sistema de financiamiento escolar a través del voucher, que ocupa más del 90\% de la educación chilena (ELACQUA, 2012), mina profundamente el capital social de las familias. James y Hofer (1987 apud FISCHEL, 2006) consideran que el capital social son las normas y los valores de una comunidad que se transmiten a los niños a través de las relaciones estables entre los adultos. El problema del sistema de voucher esque al pretender la elección de escuela competitivas entre sí, genera una movilidad residencial que socava tales relaciones sociales.

Es posible entonces plantear que la educación aporta al desarrollo de la comunidad local a través del fortalecimiento de su capital cultural y social (ONU, 2004). El capital social incluye dos dimensiones: la capacidad específica de movilización de determinados recursos por parte de un grupo y la disponibilidad de redes de relaciones sociales (COLEMAN, 1988; COLEMAN apud RAMÍREZ, 2005). Según Fischel (2006), el capital social de una escuela es específico de ésta y no es transferible a otra.

Según la CEPAL - Comisión Económica Para Latinoamérica y el Caribe (2007), el capital social da cuenta, en buena medida, de un acervo de los agentes sociales que contribuye a una sociedad más cohesionada. La cohesión social se refiere a los mecanismos de inclusión y exclusión de las sociedades, y también a cómo estos mecanismos influyen en las percepciones y conductas de las personas en una comunidad en particular (CEPAL, 2007).

El concepto de cohesión social fue utilizado por primera vez por Durkheim (2011) para plantear que en las sociedades modernas, con una mayor división social del trabajo y mayor autonomía de los sujetos, se hace necesario desarrollar lazos que permitan mantener los vínculos entre los sujetos (BARBA; COHEN, 2011). En la actualidad, el concepto de cohesión social es más abierto y se da cuenta de varios niveles de interacción social, por lo cual hemos decidido en trabajos anteriores distinguir tres niveles: uno macro-institucional, uno comunitario y uno individual (NÚÑEZ; SOLÍS; SOTO, 2014).

El nivel macro-institucional incluye los mecanismos formales que permitirán el desarrollo de la cohesión social, como la educación y el sistema educativo, puesto que facilitan la cooperación social y refuerza los contratos entre los individuos (CEPAL, 2007). En este nivel se encuentran las relaciones entre las comunidades y el Estado, lo cual les permite sentirse parte de un proyecto país (NÚÑ̃Z; SOLÍS; SOTO, 2014). En el nivel comunitario, el vínculo entre los sujetos de una comunidad se plantea como solidaridad (DURKHEIM, 2011; MALINOSKY, 1975), según el cual, ésta se genera en relación a un elemento cultural o institución que ejerce una función articuladora. De este modo, los sujetos se cohesionan en relación a un elemento simbólico o material que se significa como un articulador tanto de las prácticas cotidianas como de las otras instituciones existentes (BARBA; COHEN, 2011). Esto es coherente con la función que cumple la escuela en espacios rurales, como facilitador y aglutinador de las relaciones entre los sujetos (NÚÑEZ; SOLÍS; SOTO, 2014).

En el nivel individual, se encuentran los comportamientos, percepciones y valoraciones de los sujetos respecto del vínculo con otros 
y con la sociedad en su conjunto. En esto se destaca la valoración de los sujetos respecto de si se sienten parte, o no, de una colectividad (CEPAL, 2007; COX, SCHWARTZMAN, 2009; BARBA; COHEN, 2011) y los sentimientos de inclusión o exclusión.

En investigaciones anteriores hemos podido concluir cómo el cierre de la escuela rural afecta los distintos niveles de la cohesión social. Esto debido a que la escuela en territorios rurales cumple funciones vinculantes, que no son sustituibles por otras instituciones; ya sea porque éstas también han ido desapareciendo o porque han perdido su fuerza articuladora (NÚÑEZ; SOLÍS; SOTO, 2014).

Para contextualizar el caso, la escuela analizada se encuentra en un territorio rural costero de la zona central de Chile, cuyas principales actividades productivas eran hasta hace poco tiempo, la producción agrícola y la pesca artesanal; sumándose fuertemente en los últimos años el turismo y la práctica de deportes acuáticos. Este cambio de un número reducido de actividades productivas a una pluriactividad, ha significado una serie de transformaciones en la composición poblacional, en la organización habitacional y territorial, las cuales son posibles de ser comprendidas como parte de la dinámica social que hoy se denomina como nueva ruralidad (ROMERO, 2012). Asimismo, la migración ciudad-campo ha implicado la emergencia de un nuevo grupo de mayores recursos socioeconómicos, generándose un barrio alto en términos residenciales, y la apertura de la primera escuela particularprivada de toda la comuna.

Debido al sistema de voucher, la escuela municipal debe competir con la escuela particular-privada por la matrícula. Si bien el Nivel Socioeconómico (NSE) promedio de los estudiantes de la escuela es medio-bajo, quienes tenían un NSE más alto, optaron por cambiarse a la nueva escuela en el año en que se desarrolló la investigación, lo cual contribuyó a disminuir la matrícula de la escuela municipal y a aumentar su riesgo de cierre.
Desde la perspectiva de la nueva ruralidad, el que la producción agrícola ya no sea el único soporte de la economía rural, implica una reconsideración del desarrollo rural en cuanto a una diversificación de metas normativas, como la reducción de la pobreza, la revalorización de la cultura y su gente, la participación social y la superación de la división rural-urbana; al mismo tiempo que garantizar la viabilidad de la agricultura campesina (ROMERO, 2012). Son estos aspectos del desarrollo rural a los cuales esta investigación busca aportar.

\section{Metodología}

En esta investigación, se desarrolló una metodología de indagación cualitativa, con un enfoque etnográfico (PUJADAS; COMAS; ROCA, 2010; ROCKWELL, 2009; GUBER, 2012). Se utilizó un diseño de investigación cuasi etnográfico (IÑIGUEZ, 2004; SILVA; BURGOS, 2011), caracterizado por visitas a terreno de duración acotada, con el fin de profundizar en un evento o situación social específica (MURTAGH, 2007) como es la experiencia de un inminente cierre escolar en la comunidad estudiada. La unidad de análisis fue una escuela rural municipal ubicada en la zona central del país, que se encontraba bajo aviso de cierre, es decir, que oficialmente estaba próxima a cerrar.

Como técnica de producción de datos para el trabajo con niñas y niños, diseñamos una herramienta metodológica de carácter proyectivo y expresivo (CELENER, 2002), que incorpora tanto elementos gráficos como verbales. Si bien es cierto existe un variado abanico de técnicas en la investigación cualitativa, pocas de ellas se adaptan al trabajo con niños y niñas directamente, puesto que han sido construidos por y para adultos (RODRÍGUEZ, 2006) o considerándolos sólo como en oposición a estos (DORNER, 2014).

La adaptación realizada consistió en desarrollar una herramienta que facilitara la expresión de las niñas y niños, así como la participación de todas y todos. Esta herramienta 
corresponde a la elaboración de un dibujo colectivo que representa un mapa subjetivo de la comunidad, donde lo importante no es la recreación del pueblo de manera fiel a la realidad, sino que dé cuenta de cómo las niñas y niños lo vivencian y perciben. Así lo central es qué es lo que destacan en el mapa que elaboran, en dónde ubican la escuela dentro de él y cuáles son los circuitos por los cuales las niñas y niños circulan cotidianamente.

Se optó por el dibujo, debido que para los niños constituye una forma de comunicación interpersonal (COHEN, 2012), puesto que permite la expresión frente a temas que tienen una fuerte carga emocional para los cuales las palabras pueden no ser fáciles (PINILLA, 2006). Según Piaget (1972), el dibujo cumple una función semiótica que se encuentra entre el juego simbólico y la imagen mental. De este modo, el dibujo se ha ido desarrollando como técnica con grandes potencialidades en el trabajo con niñas y niños, pues

[...] permite expresar de manera privilegiada la proyección del esquema corporal, la imagen de sí y sus cambios a lo largo del desarrollo, como así también las capacidades, habilidades, conflictos, deseos, impulsos y ansiedades de los sujetos. (CELENER, 2002, p. 111).

Es importante señalar, que lo central en este caso no es el dibujo como dato a interpretar, sino todo el proceso de producción, incluyendo los significados desplegados y la reflexión desarrollada por las niñas y niños durante el taller (BAGNOLI, 2009).

Esta técnica del dibujo colectivo fue utilizada para comprender la significación que hacen los sujetos de la "dimensión simbólica de los procesos sociales que se construyen como problemáticos" (GUTIÉRREZ, 2011, p. 116), considerando como tal el aviso de cierre de la escuela. Ésta se basó en algunos principios del Grupo de Discusión, en tanto instancia de discusión grupal en la que los participantes enuncian significados asociados a un cierto problema o fenómeno, planteado de tal modo a producir un discurso grupal (MELLA, 2003). Por este motivo era importante que las niñas y niños desarrollaran el mapa subjetivo de manera conjunta y no de manera individual. Asimismo, lo relevante no sólo fue la producción del dibujo, sino las opiniones y comentarios que las niñas y niños daban durante su elaboración.

La introducción del mapa subjetivo buscó facilitar la expresión de opiniones y sentimientos de las niñas y niños, así como la interacción entre los miembros del grupo, algo que también se ha sugerido para los Focus Group con niños (ARAMBURUZABALA, 2000). Esta técnica grupal vino a resolver problemas en experiencias previas de esta misma investigación, cuando el equipo evaluó que el encuadre de entrevista semi-estructurada no logró facilitar adecuadamente la expresión de los niños. Nuestra hipótesis, que sirvió también para guiar este nuevo diseño, es que en la investigación con niños es fundamental pensar el lugar del investigador adulto para no reproducir una escena asimétrica donde el niño, como menor de edad, respondiera al adulto, sino que era importante generar las condiciones para que estos pudieran hablar y discutir en un espacio donde esas distancias se diluyeran. El uso del dibujo y del trabajo grupal permiten que la asimetría adulto-niño pueda horizontalizarse (VERGARA et al., 2015).

\section{Participantes}

Un elemento importante a destacar, es que la escuela a la cual las niñas y niños pertenecen es una escuela rural multigrado, es decir, una escuela pequeña en la cual todos los estudiantes se educan en una misma sala, aunque son de niveles distintos. Para efectos de este trabajo, se buscó respetar esta misma dinámica de la escuela, por lo cual participaron todos los estudiantes de la escuela, sin distinción de edad. En total eran ocho estudiantes de tercero, cuarto y quinto año básico de la escuela, 
de edades fluctuantes entre 8 y 11 años, de los cuales dos eran niñas y seis eran niños. Entre ellos se incluyó un niño de 5 años, que asistía a la escuela sin estar matriculado ${ }^{2}$.

\section{Procedimiento}

La modificación del encuadre consistió en realizar un taller, en donde se motivó la reflexión y rememoración de eventos significativos sobre la escuela y su relación con la comunidad, a través de un dibujo colectivo. Esta actividad se desarrolló dentro de la jornada escolar.

De este modo, se les solicitó a las niñas y niños que dibujaran en conjunto un mapa de su comunidad, en donde ubicaran la escuela en relación a sus casas y a los lugares que destacan de su entorno (Dibujo 1). A continuación, se les solicitó que relataran el viaje que realizan desde sus casas a la escuela, sobre lo cual se formularon preguntas que movilizaron sus reflexiones y sentimientos.

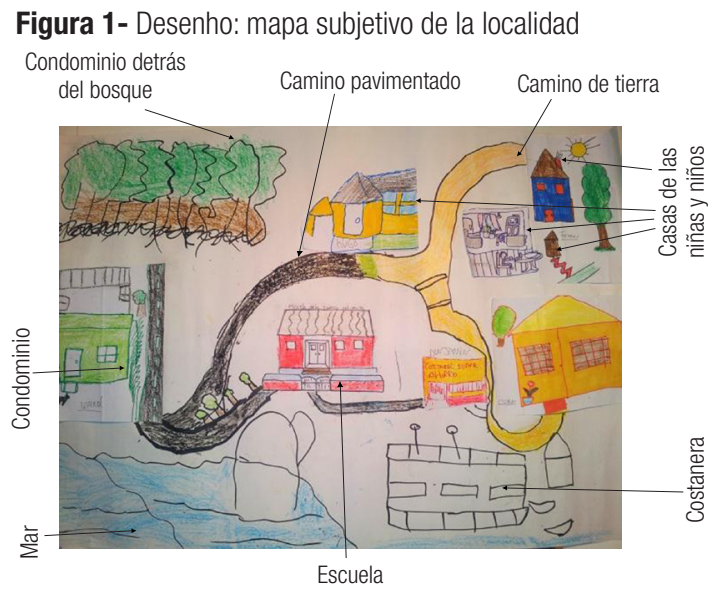

Fonte:

La estructura del taller contempló bienvenida, actividad, plenario y dinámicas de cierre de la experiencia. Se registró el audio de las conversaciones e interacciones de los niños, junto con fotografías de los diseños realizados.

2- Esta es una práctica común en zonas rurales donde no hay establecimientos de educación preescolar.
El tema del cierre de la escuela fue abordado directamente con las niñas y niños puesto que ya estaban informados de la situación. Todos los participantes firmaron una carta de Asentimiento Informado, en el que se les explicaba de manera simple los objetivos y las características de la actividad, siendo leídas por los investigadores. Asimismo, los adultos responsables de los niños y la profesora a cargo, firmaron una carta de Consentimiento Informado, con la cual daban su consentimiento para que los niños participaran. Durante todo el proceso se tomaron todos los resguardos éticos para asegurar el bienestar de las niñas y niños. Utilizamos un lenguaje inclusivo, haciendo mención a niñas y niños; sin embargo, el género no constituyó un eje de análisis para interpretar los resultados en este trabajo en particular.

\section{Análisis}

El análisis se realizó sobre los relatos e interpretaciones hechos por los estudiantes respecto a sus dibujos y sus respuestas a las preguntas realizadas por los investigadores. Al igual que todos los datos producidos, se analizó según los lineamientos de la Teoría Fundamentada, que propone producir teoría emergente a partir de un análisis apegado a los datos, los cuales se estructuran en base a categorías (STRAUSS; CORBIN, 2002). Plantea un método comparativo constante, que busca construir modelos teóricos basados en las interrelaciones de las diferentes dimensiones del fenómeno en estudio. A través de este procedimiento es posible lograr un estudio descriptivo a la vez que explicativo, mediante 3 vías de análisis: codificación abierta, que comienza con la conceptualización de los datos, realizándose una representación abstracta de un acontecimiento, acción/interacción u objeto que el investigador identifica como significativo, bajo la forma de etiquetamiento por categorías; codificación axial, que consiste en relacionar las categorías y subcategorías, según sus propiedades y dimensiones; codificación 
selectiva, en que se construyen redes de datos, volviendo a unir lo que en la fase anterior se fragmentó. En esta fase se integran y se refinan las teorías en torno al núcleo central del fenómeno. Se determina una categoría central que represente el tema nodal de investigación, aunque ninguna lo captura íntegramente.

\section{Resultados}

Como resultados del proceso, se construyeron 4 macro-categorías. Estas categorías se produjeron para todo el estudio de caso, construidas a través del análisis de entrevistas y focus-group con adultos y de talleres con niñas y niños. Los datos producidos en el taller formaron parte de cada categoría. Estas son: La escuela da vida, la comunidad nourbana: la diferencia significada como amenaza y no somos considerados: la invisibilización de la comunidad desde las politicas públicas y la profesora como articuladora de la comunidad. De las cuatro categorías, la categoría La escuela da vida, fue construida a partir de lo planteado solamente por los adultos ${ }^{3}$, pues no apareció como tal en las producciones infantiles.

\section{a) La comunidad no-urbana: la diferencia significada como amenaza:}

Los sujetos investigados no se definen a sí mismos como sujetos rurales, pero sí establecen diferencias con lo urbano. Hay para ellos una clara diferenciación entre lo urbano y lo no-urbano y esta diferencia es significada como una amenaza, como se puede observar en los fragmentos de entrevistas que transcribimos:"[E: ¿y qué pasaría si el colegio se cierra?] N2: Tendríamos que irnos a estudiar a Navidad y nos quedaría más lejos aún [...]

3- Esta parte de la investigación se encuentra publicada en La escuela da vida: el cierre de escuelas rurales en Chile según las comunidades (NUÑEZ et al., 2013). En ella se concluye que para la comunidad la escuela es un motor de desarrollo y su cierre es experimentado como un signo de muerte social.
N3: sería más fome ${ }^{4}[\ldots]$ y en $\mathrm{Navidad}^{5}$ hay más accidentes”. (G.D.Es $1^{6}$ ).

El eventual suceso de cierre de la escuela del sector se constituye como una amenaza frente a este otro externo que debe ser enfrentado, esta vez, por separado. Esto es experimentado desde los niños como la presencia latente de un eventual peligro que se encuentra en las afueras de su localidad y que deberán enfrentar en caso de que su escuela se cierre. Los niños deben desplazarse desde su sector para llegar a la escuela (Dibujo 1), pero en el caso de cierre, ir a estudiar en el pueblo implicaría desplazarse más lejos aún. De este modo, valoran la cercanía de la escuela, que en su dibujo aparece protagónica en el centro, y atribuyen mayor seguridad al hecho de estudiar en su localidad más pequeña. A diferencia de esto, estudiar en el pueblo se asume como algo riesgoso por la posibilidad de accidentes. Este peligro es mayor cuando además, como veremos, implica que debe ser enfrentado por cada estudiante por separado.

[E: ¿Y qué pasaría si el colegio se cierra?] N3: Sería fome [...] N6: Sí, porque ahí uno no conoce a nadie y por ejemplo a uno lo ponen en San Antonio, a otro en otro lugar y no estamos juntos como aquí, que nos conocemos todos casi [¿Les gusta estar todos juntos] Todos: ¡Sí! (G.D.Es1).

[...] N5: Si, a mí no me gustaría que cierre [E: ¿Por qué?] N1: No, es que después vamos a tener que ir para otro lado, $\mathrm{y}$ vamos a estar todos separados, N6: Sí, y uno en otra escuela no conoce a casi nadie (G.D.Es1).

Los estudiantes mencionan de manera efusiva los elementos afectivos asociados a dejar la escuela. Tal como en su colorido

4 - Fome en Chile puede tener varios significados, generalmente significa algo aburrido pero también puede usarse para describir algo desagradable. 5- Pueblo cercano a la comunidad investigada.

6- Fragmento de Grupo de Discusión con Estudiantes no 1 - G. D. Es1. 
dibujo donde la escuela brilla en el centro en color rojo, se expresan de manera vehemente respecto de este punto. Existe una preocupación por el vínculo que han generado como grupo de pares, enfocando las consecuencias negativas del cierre sobre el hecho que serán separados y podrían encontrarse en diferentes establecimientos, lo que pondría en riesgo dicho lazo. Para los estudiantes el cierre supone un peligro, puesto que amenaza la relación afectiva que se construye en su interacción cotidiana e implica ingresar a una nueva escuela sin redes sociales previas. Para las niñas y niños parte importante de la experiencia escolar constituye el sentirse pertenecientes a un grupo socialmente cohesionado.

Debido a que la escuela es multigrado, todas las niñas y niños comparten una misma aula, también almuerzan juntos en el comedor y suelen jugar juntos en el patio. Estar todos juntos es la experiencia escolar característica en escuelas rurales multigrado, experiencia que temen perder al cerrarse la escuela y dispersarse en otras. Efectivamente, cuando las escuelas multigrado se cierran, por lo general los niños son trasladados a otras escuelas con un curso por nivel, por lo que la experiencia del estar todos juntos es muy probable que no vuelva a repetirse.

La forma colectiva en que se construyó el dibujo también fue considerada dentro de esta categoría, pues dio cuenta de cómo el grupo funciona. Todos participaron en la elaboración del dibujo, sin distinción de género ni edad, estableciéndose una dinámica colaborativa entre los niños, que fue facilitada por los entrevistadores de manera muy fluida. Atribuimos que dicha dinámica grupal responde a la costumbre de las niñas y niños de trabajar bajo la modalidad de aula multigrado, lo cual reafırma lo que los niños dicen de su escuela, pues es posible de constatarlo a través de las observaciones de campo.

Cabe señalar que en Chile existe libertad de elección por parte de los padres del establecimiento en el que matriculan a sus hijos, a ello se refiere uno de los estudiantes cuando señala: "por ejemplo a uno lo ponen en San Antonio, a otro en otro lugar”. Esto hace que si la escuela se cierra, no todos los niños serán trasladados a una misma escuela, necesariamente.

De este modo, la escuela rural es para las niñas y niños una institución que los cohesiona $\mathrm{y}$ los mantiene unidos como comunidad escolar. Esto corresponde al nivel comunitario de la cohesión social y el cierre de la escuela lo afectará directamente. A su vez, las niñas y niños reconocen en la escuela el valor de contar con redes sociales, que desaparecerían en otra escuela. Esto confirma los resultados de otras investigaciones que muestran cómo el capital social que consiste en las redes sociales que la escuela genera, es específico de la escuela y no es transferible a otra (FISCHEL, 2006). Esto, según el propio Fischel es relevante a nivel político, ya que estas relaciones sociales generan lazos que permitirían la organización política, poniendo como ejemplo, lo mismo que los niños mencionan, una comunidad organizada puede luchar mejor contra las amenazas del medio, como una esquina mal señalizada, por ejemplo.

\section{b) No somos considerados: la invisibilización de la comunidad desde las politicas públicas}

Los actores locales significan cambios importantes en su sector en los últimos años, los cuales se acompañan de un sentimiento de malestar, puesto que han implicado transformaciones en sus vidas cotidianas. Lo interesante de esta categoría, es que muestra que para las niñas y niños el cierre de la escuela se relaciona de alguna manera con otros cambios sociales, así como la forma en que experimentan las dinámicas de la nueva ruralidad. Los relatos emergen en gran medida a partir de la elaboración del mapa subjetivo (Dibujo 1), en donde los estudiantes han ubicado puntos significativos para ellos en la localidad. Nótese cómo han dividido el camino entre la parte pavimentada y el camino de tierra, que 
es donde las niñas y niños ubican sus propias casas. "N3: [me gusta Blanes ${ }^{7}$ ] porque es tranquilo, me gusta porque es tranquilo, pero cuando hay fin de semana largo, es medio fome, porque pasan puros autos y no podemos salir a andar en bicicleta." (G.D.Es1).

Las niñas y niños, valoran la tranquilidad del lugar como algo positivo; sin embargo, durante ciertos períodos ven impedido su libre desplazamiento por el sector. La calle es poblada por los adultos que manejan en vehículos, foráneos en su gran mayoría. Desde las impresiones de los estudiantes, se puede apreciar un malestar respecto de la brecha social y material percibida entre ellos y los nuevos habitantes que están poblando el sector, en algunos casos esto es vivenciado como una imposibilidad en sus prácticas cotidianas (como por ejemplo, salir a jugar a la calle), y en otros casos, como veremos, como una explícita diferenciación de clase. Aquí es aclaratorio mencionar que en las zonas rurales los campesinos han reemplazado el caballo por la bicicleta, siendo éste uno de los medios de transporte más utilizados en la zona del estudio.

N5: Blanes creció por los surfistas, por eso hicieron la costanera. E1: ¿por qué dicen que creció con los surfistas? N5: porque la municipalidad así, a veces sólo arregla el camino sólo hasta el camping, a veces para arriba y no arregla para más arriba. E2: ¿y por qué crees tú que pasa eso? N5: no sé, porque a veces cuando, a veces algunas mamás han reclamado que coloquen pavimento en Manzanilla y dijeron que iban a colocar y nunca han colocado, todas las mamás reclamaron, nunca han colocado. N1: colocan en un poquito no más, en una mitad de allá, así poquitito pero eso no es na' (G.D.Es1).

En el dibujo, el camino pavimentado, a pesar de que en la realidad es más largo y central,

7- Blanes y Manzanilla son nombre de fantasía que se han creado para mantener el anonimato de las y los participantes. aparece ocupando un lugar muy constreñido dentro del conjunto. En la discusión, a través de la división entre el camino pavimentado y el camino de tierra, los estudiantes significan con claridad la posición desigual en la cual se sitúa la comunidad respecto a los nuevos habitantes y el lugar que ocupa el municipio en las transformaciones del sector; atributos principales de esta categoría. Los niños saben que es el Municipio el que toma ciertas decisiones que son importantes para la vida cotidiana de la localidad y han construido su perspectiva acerca de ellas. Atribuyen a la llegada de los surfistas la preocupación del Municipio por arreglar ciertos tramos del sector ("sólo hasta el camping”) y la razón del crecimiento de Blanes, lo cual ven reflejado en la construcción de la costanera $^{8}$, una obra recientemente inaugurada.

Desde las niñas y niños, la figura municipal desconoce las necesidades de sus familias, privilegiando los intereses de foráneos por sobre los suyos. Esto a pesar del reclamo insistente por parte de sus madres, quienes aparecen como las principales interlocutoras frente al Municipio. Ante el reclamo de sus madres, el municipio parece entregar una respuesta dudosa para los niños: promete algo que luego no cumple, o responde, pero de manera insuficiente en relación a lo demandado.

De este modo las niñas y niños sienten que ellos y sus familias se encuentran marginados de las decisiones que toma el Municipio. Esto afecta la cohesión social en distintos niveles. En términos macro- institucionales, el Municipio es percibido como una institución que privilegia a algunos en desmedro de otros y que el progreso alcanzado está dirigido a ellos, quedando la comunidad marginada de sus beneficios. A nivel comunitario, las niñas y niños denotan una fragmentación entre "los surfistas" y ellos y sus familias. Estos son identificados como grupos diferentes y desiguales, puesto que los intereses de los primeros priman por sobre los segundos. Finalmente, a nivel individual, las niñas y niños

8- La Costanera es una construcción a orillas del mar con fines turísticos. 
sienten cómo estas transformaciones afectan su mundo infantil y lo restringen ("no podemos salir a andar en bicicleta”), generando malestar.

\section{c) El profesor como articulador de la comunidad}

Para los participantes adultos, la figura del profesor representa uno de los principales articuladores de la comunidad, pues es quien organiza una serie de encuentros entre los habitantes del pueblo. Por su parte, para las niñas y niños el lugar protagónico de la profesora se encuentra dado por la relación pedagógica y la enseñanza para el aprendizaje, siendo quien articula la comunidad escolar.

E1 ¿Qué cambiaría con el cierre de esta escuela para ustedes? N6: No nos pueden enseñar como acá... E1 ¿Cómo es eso? N6: Porque acá a veces nos enseñan, y cuando no aprendemos nos vuelven a enseñar de nuevo... y a veces nos enseñan con chistes... Todos: ¡Sí! (G.D.Es1).

N5: sí, acá la tía nos vuelve a leer, cuando no entendemos nos vuelve a decir cómo es... (G.D.Es1).

Lo expresado por los participantes nos revela la importancia de la relación que se establece entre la profesora y las niñas y niños; relación que es exclusiva de esa escuela e irremplazable por otra. Lo que está puesto al centro, es la relación pedagógica que se establece en torno al aprendizaje: cuando alguien no aprende, el profesor vuelve a enseñar. Para las niñas y niños el aprendizaje no es una simple reacción inmediata a la enseñanza de la profesora, sino que requiere su reiteración sucesiva hasta conseguirlo. Existe además la aprehensión de que dicha forma de enseñanza basada en la reiteración no se dé en otra escuela ("No nos pueden enseñar como acá") y que por lo tanto, el aprendizaje no pueda ser asegurado en otro contexto.

Junto a la reiteración de la enseñanza, el uso de los chistes es reconocido por las niñas y niños como otro recurso pedagógico utilizado por la profesora y lo valoran positivamente como algo que hace placentera la experiencia escolar. De esta forma, la profesora se establece como figura central en el aprendizaje de los niños y la exclusividad de la relación pedagógica propia de esta escuela se constituye como uno de los atributos de esta categoría.

\section{Conclusiones}

Los resultados muestran cómo el eventual cierre de la escuela trae consecuencias para la cohesión social en sus distintos niveles, no sólo para los adultos, sino también desde la visión de las niñas y niños. Esto resulta particularmente preocupante, por cuanto las escuelas rurales en Chile reciben a los sectores de mayor pobreza ${ }^{9}$, quienes son los más afectados en términos de desarrollo social cuando la escuela se cierra. Los sujetos, tanto adultos como niños, se sienten marginados de un proyecto país que parece no considerar sus demandas.

La evidente segregación educacional chilena es también vivida por los niños, tanto como sujetos de un territorio determinado, como parte de una clase social afectada por la llegada de nuevos habitantes. Además, la infancia como grupo social generacional debe aceptar vivir en ciertas condiciones de relación con el mundo adulto que pueden terminar por segregarlos aún más. No obstante, en este caso, también es posible apreciar las particularidades de la escuela rural multigrado en términos de cohesión social. Ésta congrega a los niños en un sólo grupo, sin establecer separaciones por edad, contrario a lo que generalmente sucede en la escuela urbana. Esto permite a las niñas y niños desarrollar una experiencia escolar donde prima la vivencia de lo común, sintiéndose miembros de una comunidad. Es destacable cómo los niños y niñas dan cuenta de una comunidad conformada por ellos, sus padres, madres, municipalidad, la profesora. La importancia que

9 - La indigencia en sectores rurales tiene una incidencia de un 3,5\% (CHILE, 2008). 
le dan al otro, tanto en la entrega de recursos como en los aspectos amenazantes que encarna, dan cuenta del lugar de los niños como agentes sociales en sus comunidades, hábiles analistas de sus realidades. Las niñas y niños han ido construyendo su propia visión respecto de las transformaciones de su localidad, de las racionalidades que las sustentan y el lugar del Municipio en ellas. En este contexto, el cierre de la escuela parece corresponder a una de estas transformaciones.

Es importante destacar que esta experiencia de lo común y el sentirse parte de una comunidad no es transferible a otra escuela, tal como lo expresan las niñas y niños. Esto contradice los planteamientos en la base de la fusión y cierre de escuelas ${ }^{10}$, puesto que trasladar niños de una escuela a otra implica la pérdida de un capital social acumulado. Hasta ahora el énfasis se ha puesto en mantener el derecho a la educación en términos de acceso al momento de los cierres, asegurando la matrícula y el transporte de las niñas y niños; sin embargo, para ellas y ellos la calidad de los aprendizajes y el capital social no están asegurados con el traslado.

Consideramos que la modificación del encuadre, consistente en introducir técnicas de facilitación grupal y la introducción del dibujo colectivo para el trabajo con niños y niñas, resultó una herramienta útil en tanto posibilitó la participación de los estudiantes en la investigación. Creemos que las significaciones de los estudiantes les otorgaron una tonalidad particular a las categorías de análisis construidas, ya que provienen de una experiencia desarrollada en tanto actores de su comunidad y partícipes de un relato construido comunitariamente desde un lugar pocas veces visitado en la investigación social, como lo es la infancia. Asimismo, consideramos que las producciones simbólicas de los niños no son significaciones

10- La fusión de escuelas corresponde a una iniciativa que ha sido implementada en países como Estados Unidos, conocida como school consolidation. Consiste en fusionar dos o más escuelas en una sola que agrupe a todos los estudiantes, permitiendo un gasto fiscal más eficiente y mayor diversidad curricular. especiales ni distintas. Nuestra perspectiva no considera a los niños menores en términos de su producción simbólica. Sus producciones también se constituyen socialmente, en culturas situadas. No son simples repetidores de lo que los adultos dicen, y tal como vimos, integran las opiniones de los adultos significativos pero con el análisis de sus propias experiencias. Su lugar social, no obstante, suele estar mermado por el hecho de ser considerados menores, y más aún, siendo parte de las silenciadas comunidades rurales del país.

Las diferencias sociales y la desigualdad que afectan a la comunidad no es solamente un tema de adultos. Las niñas y niños también construyen su percepción acerca de ello en lo que les toca en sus subjetividades y prácticas sociales: la pérdida de sus espacios de desplazamiento libre y autónomo, la diferencia en las condiciones materiales de vida y cómo el reclamo de sus madres no es suficiente para equipararla; los cambios en la infraestructura y urbanización de la localidad. Todas ellas transformaciones que los niños atribuyen al ajuste del Municipio hacia las necesidades de otros, que a pesar de ser nuevos en el sector, resultan ser prioritarios.

Estos últimos, son aspectos interesantes de analizar en las dinámicas sociales emergentes que caracterizan la nueva ruralidad. Los límites entre lo rural y lo urbano parecen hacerse más difusos, los nuevos fenómenos migratorios desde la ciudad al campo traen aparejados el arribo a lo rural de formas de organización social y territorial más propias de lo urbano, como la segregación residencial y escolar, la competencia entre escuelas. Todas estas transformaciones vistas, en este caso, desde la perspectiva de las niñas y niños rurales.

El cierre masivo de escuelas rurales en Chile da cuenta de la falta de una política de desarrollo rural, que trasciende el ámbito educativo; puesto que la escuela, al ocupar un lugar central en la dinámica social, impacta con su cierre en distintas dimensiones. Las metas del desarrollo rural propuestas por la perspectiva de la nueva ruralidad, como la revalorización de la cultura y 
su gente, la participación social y la superación de la división rural- urbana, no están siendo consideradas dentro de la toma de decisiones.

En cuanto al trabajo con niñas y niños, es importante destacar que la inclusión de sus relatos implica variados desafíos. Por un parte, los descritos por Castro, Ezquerra y Argos (2011) acerca de la necesidad de integrar a todos los participantes, en especial a aquellos que por motivos varios se restan de la actividad, así como la de focalización de la conversación en las temáticas que se plantean como centrales por el grupo por sobre las conversaciones anexas, etc. Sin embargo, también creemos necesaria: 1) la búsqueda permanente de actividades facilitadoras de la conversación de todos los niños ya que la conversación puede girar sólo en torno a los participantes más extrovertidos (problema que Parker (2007), ha detectado y analizado en el trabajo de la psicología comunitaria); 2) considerar la discusión sobre la pertinencia de incluir análisis interpretativos de los dibujos, en tanto materiales proyectivos, implica un tipo de análisis que compartiría fronteras con líneas teóricas cercanas a otras disciplinas, por ejemplo el Psicoanálisis, la Estética, que creemos que pueden complementar los datos construidos en el proceso de discusión grupal; 3) incluir el análisis de las interacciones grupales en el proceso de construcción del dibujo colectivo, como parte informante de un modo particular de producción de los datos que dado el caso, brindaría coordenadas que enriquecen el proceso de análisis y dan más luces en relación a las interacciones entre pares y cómo esto genera respuestas que podrían verse enriquecidas por la colaboración.

La Convención de los Derechos de la Infancia de las Naciones Unidas (UNICEF, 2006) consolida el derecho del niño de expresar su opinión y ser escuchado por los adultos. Si bien esta convención puede ser tomada como una mera declaración de principios, creemos que la posibilidad de incluir la voz de los niños, en tanto estudiantes, actores de su comunidad y sujetos de derecho, es completamente posible e incluso necesaria si se pretende investigar en profundidad un fenómeno que afecta -como el caso en estudio- a una escuela, una comunidad y al mismo tiempo un país, al transformarse el cierre de escuelas rurales en un fenómeno masivo. De tal modo, la inclusión de los niños y niñas debiera considerarse como una obligación ética si es que se considera a la infancia en tanto posición subjetiva diferente de la adulta, no anterior o menor a ésta, sino una que comporta una visión particular de los fenómenos sociales que afectan a una comunidad de la cual son miembros.

\section{Referencias}

ARAMBURUZABALA, Pilar; PASTOR, Ana. Grupos de discusión con niños: un proyecto Europeo del asma infantil. Psicothema, Oviedro, v. 12, n. 2, p. 39-41, 2000.

BAGNOLI, Anna. Beyond the standard interview: the use of graphic elicitation and arts-based methods. Qualitative Research, Cardiff, v. 9, n. 5, p. 547-570, 2009. Disponible en: <D0I: 10.1177/1468794109343625>. Acceso en: 13 abr. 2015.

BARBA, Carlos; COHEN, Néstor (Comp.) Perspectivas críticas sobre la cohesión social: desigualdad y tentativas fallidas de integración social en América Latina. Buenos Aires: Clacso, 2011. Disponible en: <http://biblioteca.clacso.edu.ar/ar/libros/ clacso/crop/BarbaSolano-Cohen.pdf>. Acceso en: 05 mar. 2013.

CASTRO Ana; EZQUERRA Pilar; ARGOS Javier. Dando voz y protagonismo a la infancia en los procesos de investigación e innovación educativos. Revista Fuentes, Sevilla, n. 11, p. 107-123, 2011.

CELENER, Graciela (Coord.) Técnicas proyectivas: actualización e interpretación en los ámbitos clínico, laboral y forense. Buenos Aires: Lugar, 2002. 
CEPAL. Comisión Económica para Latinoamérica y el Caribe. Cohesión social: inclusión y sentido de pertenencia en América Latina y el Caribe. Santiago: Naciones Unidas, 2007. Disponible en: <http://www.oei.es/quipu/cohesion_socialAL_CEPAL.pdf>. Acceso en: 18 dic. 2014.

CHILE. Ministerio de Hacienda. Programas de servicios de fomento a sectores especiales: Programa de Desarrollo Local (Prodesal) y Programa de Desarrollo Comunas Pobres (Prodecop). Santiago: [s. n.], 2008.

COHEN, Silvia. Del garabato al dibujo: una mirada diacrónica del dibujo infantil. Ficha de cátedra, Facultad de Psicología, Universidad Nacional de Tucumán, 2012. Disponible en: <http://www.psicologia.unt.edu.ar/index.php?option=com_docman\&task=doc_ download\&gid=508\&ltemid=248. $>$. Acceso en: 30 nov. 2014.

COLEMAN, James. Social capital in the creation of human capital. The American Journal of Sociology, Chicago, v. 94, p. S95-S120, 1988.

CORNEJO, Rodrigo. El experimento educativo chileno 20 años después: una mirada crítica a los logros y falencias del sistema escolar. Revista Electrónica Iberoamericana sobre Calidad, Eficacia y Cambio en Educación, Madrid, v. 4, n. 1, p. 118-129, 2006.

COX, Cristian; SCHWARTZMAN, Simon (Ed.). Políticas educativas y la cohesión social en América Latina. Santiago de Chile: Uqbar, 2009.

DORNER, Lisa. From relating to (re)presenting: challenges and lessons learned from an ethnographic study with young children. Qualitative Inquiry, London, p. 1-12, 30 dic. 2014. Disponible en: < DOl: 10.1177/1077800414557824>. Acceso en: 10 mar. 2015.

Durkheim, Émile [1897]. De la division du travail social. Paris: Presses Universitaires de France, 2011. (Collection bibliothèque de philosophie contemporaine).

ELACQUA, Gregory. The impact of school choice and public policy on segregation: evidence from Chile. International Journal of Education Development, Amsterdam, v. 32, n. 3, p. 444-453, 2012. Disponible en: <D01:10.1016/j.ijedudev.2011.08.003>. Acceso en: 3 mar. 2013.

FISCHEL, William. Why voters veto vouchers: public schools and community-specific social capital. Economics of Governance, Berlin, v. 7, n. 2, p. 109-132, 2006

GUBER, Rosana. La Etnografía. Buenos Aires: Siglo XXI, 2012.

GUTIÉRREZ, Jesús. Grupo de discusión: ¿prolongación, variación o ruptura con el focus group? Revista Cinta Moebio, Santiago, n. 41, p. 105-122, 2011. Disponible en: <www.moebio.uchile.cl/41/ gutierrez.html>. Acceso en: 5 jun. 2012.

HSEIH, Chang-Tai; URQUIOLA, Miguel. When school competes, how they compete? An assessment of Chile's nation wide school voucher program. Washington: World Bank's Development Group, 2002.

IÑIGUEZ, Lupicinio. Métodos cualitativos: extractos de métodos cualitativos en psicología social. Revista de Psicología Social Aplicada, Valencia, v. 5, n. 1-2, p. 5-26, 2004.

JAMES, Allison; JAMES., Adrian. Constructing childhood: theory, police and social practice. London: Palgrave Macmillan, 2004.

JAMES, Allison; JAMES, Adrian. Key concepts in childhood studies. London: Sage, 2008.

MALINOWSKI, Bronislav [1922]. Los argonautas del Pacífico Occidental: un estudio sobre comercio y aventura entre los indígenas de los archipiélagos de la Nueva Guinea Melanésica. Barcelona: Península, 1975.

MELLA, Orlando. Metodología cualitativa en ciencias sociales y educación. Santiago: Primus, 2003.

MURTAGH, Lisa. Implementing a critically quasi-ethnographic approach. The Qualitative Report, E.E.U.U., v. 12, n. 2, p. 193-215, 2007. Disponible en: <www.nova.edu/ssss/QR/QR12-2/murtagh.pdf>. Acceso en: 21 jul. 2013.

NúÑEZ, Carmen Gloria et al. La escuela da vida: el cierre de escuelas rurales en Chile según las comunidades. Revista: Sociedad Hoy, Concepción, v. 24, p. 49-54, 2013. 
NúÑEZ, Carmen Gloria; SOLÍS, Camila; SOTO, Rodrigo ¿Qué sucede en las comunidades cuando se cierra la escuela rural? Un análisis psicosocial de la política de cierre de las escuelas rurales en Chile. Revista Universitas Psychologica, Bogotá, v. 13, n. 2, p. 615-625, 2014. Disponible em: <D01:10.11144/Javeriana.UPSY13-2.qscc>. Acceso en: 7 dic. 2014

ONU. Organización de las Naciones Unidas para la Educación, la Ciencia y la Cultura. Organización de las Naciones Unidas para la Alimentación y la Agricultura. Educación para la población rural en Brasil, Chile, Colombia, Honduras, México, Paraguay y Perú. Roma: CIDE: 2004.

PARKER, lan. La deconstrucción de la psicopatología en la investigación-acción. Archipiélago, Madrid, n. 76, p.65-74, 2007.

PIAGET, Jean. Memoria e inteligencia. Buenos Aires: El Ateneo, 1972.

PINILLA, Marcela. Las representaciones gráficas de niños como metodología de investigación en un contexto rural de violencia armada en Colombia. Revista Chilena de Antropología Visual, Santiago, n. 8, p. 143-156, 2006.

PUJADAS, Joan; COMAS, Dolors; ROCA, Jordi (Coord.). Etnografía. Barcelona: UOC, 2010.

RAMÍREZ, Jorge. Tres visiones sobre capital social: Bourdieu, Coleman y Putnam. Acta Republicana Política y Sociedad, Guadalajara, v. 4, n. 4, p. 21-36, 2005.

ROCKWELL, Elsa. La experiencia etnográfica: historia y cultura en los procesos educativos. Buenos Aires: Paidós, 2009.

ROMERO, Juan. Lo rural y la ruralidad en América Latina: categorías conceptuales en debate. Revista Psicoperspectivas, Valparaíso, v. 11, n. 1, p. 8-31, 2012.

RODRÍGUEZ, Iván. Redefiniendo el trabajo metodológico cualitativo con niños: el uso de la entrevista de grupo aplicada al estudio de la tecnología. Empiria, Madrid, n. 12, p. 68-88, 2006.

SILVA, Carlos; BURGOS, César. Tiempo mínimo-conocimiento suficiente: la cuasi-etnografía sociotécnica en psicología social. Psicoperspectivas, Valparaíso, v. 10, n. 2, p. 87-108, 2011. Disponible en: <D0I:10.5027/PSICOPERSPECTIVAS-VOL10-ISSUE2FULLTEXT-146>. Acceso en: 10 mar. 2014.

STRAUSS, Anselm; CORBIN, Juliet. Bases de la investigación cualitativa: técnicas y procedimientos para desarrollar una teoría fundamentada. Medellín: Universidad de Antioquia, 2002.

UNICEF. Fondo de las Naciones Unidas para la Infancia. Convención de los derechos del niño. Madrid: UNICEF/Comité Español, 2006.

VERGARA, Ana et al. Los niños como sujetos sociales: el aporte de los nuevos estudios sociales de la infancia y el análisis crítico del discurso. Psicoperspectivas, Valparaíso, v. 14, n. 1, p. 55-65, ene. 2015. Disponible en: <http://www.scielo.cl/scielo. php?script=sci_arttext\&pid=S0718-69242015000100006\&lng=es\&nrm=iso>. Acceso en: 1 jul. 2015.

Recibido en: 21.07.2015

Aprobado en: 16.02 .2016

Carmen Gloria Núñez es psicóloga y doctora en ciencias de la educación. Es profesora Escuela de Psicología Pontificia Universidad Católica de Valparaíso.

Mónica Peña es psicóloga y doctora en ciencias de la educación. Escuela de Psicología Universidad Diego Portales.

Francisco Cubillos es psicólogo por la Escuela de Psicología Pontificia Universidad Católica de Valparaíso.

Héctor Solorza es psicólogo por la Escuela de Psicología Pontificia Universidad Católica de Valparaíso. 